\title{
Bullet-proof armour and hydrogen sieve add to graphene's promise
}

\section{One-atom-thick material blocks 'bullet' strikes but allows protons to pass through.}

\section{Richard Van Noorden}

27 November 2014

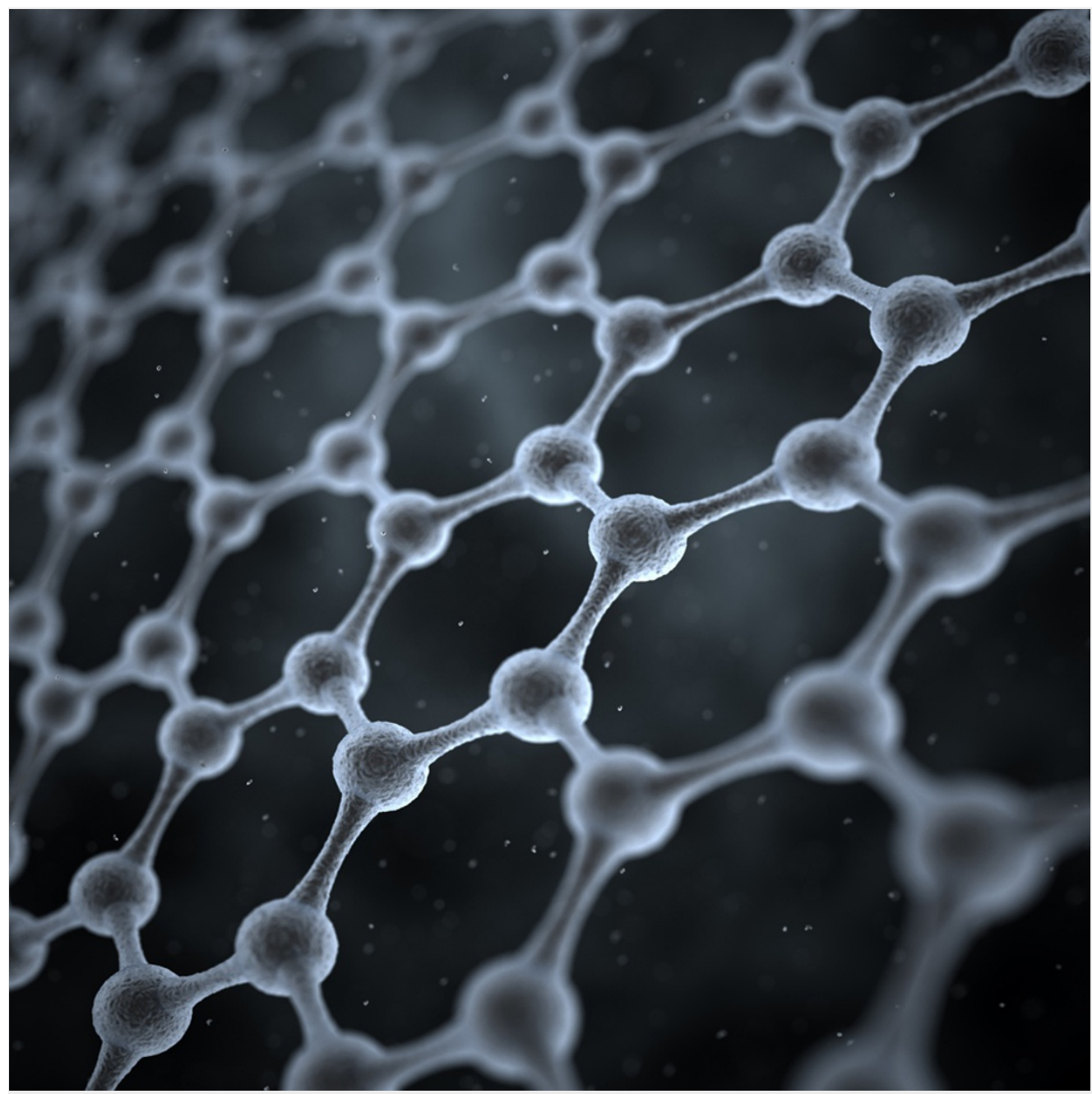

Andrey Prokhorov/Getty

The world's thinnest, strongest material — graphene - was first isolated a decade ago, but the single-atom-thick sheet of carbon is still turning up surprises.

Thought to be an impermeable barrier, research reported in Nature ${ }^{1}$ this week shows that graphene in fact allows protons to pass through it, opening up the possibility of its use as an ultrathin membrane in fuel cells. Meanwhile, a separate report in $S c i e n c e^{2}$ today reveals that graphene outperforms both steel and a composite Kevlar armour in its ability to withstand 'bullets'.

\section{Proton filter}

Protons' ability to travel through graphene suggests that the material could be used as a membrane to sieve hydrogen from air, and to help extract energy from that hydrogen in a fuel cell, says co-author Andre Geim, a materials scientist at the University of Manchester, UK, who won a Nobel prize in 2010 for his pioneering experiments on graphene.

Fuel cells convert the chemical energy stored in hydrogen (or other fuels) into electricity by breaking it apart into protons and electrons: the electrons race around an outside wire to create a current, with the protons flowing through a membrane within the cell. (Electrons and protons recombine at an electrode to react with oxygen.) Today's membranes, such as a commercial polymer called 
Nafion, are tens of micrometres thick but do not entirely prevent hydrogen fuel from leaking through; their thickness also means that proton flow is not as great as it might be, reducing the cell's power. A strong, ultrathin barrier that blocks everything except protons could solve both problems at a stroke. This, says Geim, is what graphene could provide - as could, the team reports, another atomically thin sheet made of boron nitride.

Fuel-cell experts say that the work is proof of principle, but are cautious about its immediate application. Factors such as to how grow a sufficiently clean, large graphene sheet, and its cost and lifetime, would have to be taken into account. "It may or may not be a better membrane for a fuel cell," says Andrew Herring, a chemical engineer at the Colorado School of Mines in Golden.

Geim's team used a similar approach to filter clean hydrogen from water using a graphene membrane. Such a technique might pull hydrogen from air, Geim says. "It's speculation, but before this paper, it would be science fiction," he says. Graphene itself might not be the only solution, however. Last year, researchers showed how a stacked series of graphene oxide membranes created small channels that sieved hydrogen from mixtures of carbon dioxide or nitrogen ${ }^{3}$.

\section{Strong armour}

Graphene has already been proved to be the world's strongest material — by measuring the resistance of the sheet to being pressed with a diamond tip. But now the material has been targeted by 'bullets' for the first time, with tiny silica spheres being fired at layers of graphene.

These layers can very rapidly disperse the energy of an impact before they shatter, because disruptions ripple outward through the material so fast, explains co-author Edwin Thomas, from Rice University in Houston, Texas. The upper limit on this effect is the speed of sound in a material, and in stiff, light graphene, a sound wave can travel at 22 kilometres per second — as opposed to just 332 metres per second in air. A composite material based on graphene layers and other lightweight, strong materials could be a "very promising armour system", Thomas says.

\section{A question of scale}

These latest experiments suggest even more potential for graphene, which is already being trialled for hundreds of uses, including batteries, night-vision goggles, medical scanners, condoms and photodetectors. But in all cases, industrial partners' main concern is manufacturing: whether graphene sheets, or composite materials that include graphene, can be made in large scale at sufficient quality and to reliable standards. "There is a confused market out there," says James Baker, business director of the National Graphene Institute in Manchester. One touted application - replacing the rare compound indium tin oxide used in touchscreens for mobile phones - now looks less likely, because graphene cannot provide the same quality at the same cost, for example.

Dozens of companies are currently actively making and commercializing graphene ${ }^{4}$ or graphene-based materials, but few have reached the market after years of development. But what may seem a slow pace of development to outsiders is only to be expected for new materials, says Baker. "You won't know if particular applications will work unless you talk to industry," he adds.

Nature | doi:10.1038/nature.2014.16425

\section{References}

1. Hu, S. et al, Nature http://dx.doi.org/10.1038/nature14015 (2014)

2. Lee, J.-H., Loya, P. E., Lou, J. \& Thomas, E. L. Science 346, 1092-1096 (2014).

3. Li, H. et al. Science 342, 95-98 (2013).

4. Zurutuza, A. \& Marinelli, C. Nature Nanotechnol. 9, 730-734 (2014). 